\title{
ADAPTAÇÃO DE UM MODELO DE RELATÓRIO GERENCIAL PARA AVALIAÇÃO DE SERVIÇOS HOSPITALARES
}

\author{
Guiomar Terezinha de Carvalho Aranha ${ }^{1}$ \\ Mirtes Loeschner Leichsenring ${ }^{2}$ \\ José Carlos Folegatti
}

\section{RESUMO*}

Estudo descritivo de um modelo de Relatório Gerencial, que foi elaborado para a área de Lavanderia do Hospital de Clínicas (HC) da UNICAMP com a finalidade de avaliar o desempenho das atividades realizadas por estas áreas. Para tal consecução foram aplicados os instrumentos de gerenciamento, a partir de técnicas estatísticas, com base no modelo de Gauthier e nas idéias preconizadas por Deming e Sink, com vistas a garantir a eficácia do sistema de informações, através de indicadores que permitam a mensuração e controle, para a melhoria do desempenho desses serviços hospitalares. Levando em consideração a especificidade do serviço, foi levantada, a produção total e elaboradas as planilhas: "perfil de máquinas" e "controle de roupas por período de uso" para a Lavanderia. Buscando sistematizar as informações, foi criado um sintético "manual de orientação" com as normas para preenchimento dos relatórios. Este estudo possibilitou conhecer melhor a realidade das áreas abordadas, detectar problemas ou necessidades e contribuir para a avaliação gerencial. Considera-se que este modelo poderá fornecer elementos que subsidiem a tomada de decisões para a melhoria e aproveitamento dos recursos humanos e materiais, no desenvolver das atividades administrativas das chefias e pela Administração do Hospital.

PALAVRAS CHAVES: Indicadores de Saúde, Relatório Gerencial, Estatísticas de Saúde

${ }^{1}$ Estatística responsável pelo Serviço de Estatística do HC/UNICAMP.

${ }^{2}$ Enfermeira responsável pelo Serviço de Lavanderia do HC/UNICAMP.

${ }^{3}$ Assessor Administrativo do HC/UNICAMP.

${ }^{4}$ Participação especial da Docente de Administração do Departamento de Enfermagem da FCM/UNICAMP, Creusa Guimarães Madeira. 


\section{INTRODUÇÃO}

O artigo é um estudo descritivo fornecendo elementos para análise de desempenho das diversas áreas hospitalares Após pesquisa bibliográfica exaustiva e entendimentos junto à área envolvida, Lavanderia, chegou-se a um consenso do que deveria ser um relatório gerencial, que abarcasse indicadores que medissem a melhoria do desempenho dos serviços prestados pelo Hospital. Para tal consecução foram aplicados os instrumentos de gerenciamento, a partir de técnicas estatísticas, com base no modelo de Gauthier et al (1976) e nas idéias preconizadas por Deming (1990), Sink et al (1993), com vistas a garantir a eficácia do sistema de informações, através de indicadores que permitissem a mensuração, contrôle e melhoria contínua da prestação dos serviços hospitalares.

Esse estudo de um modelo de Relatório Gerencial teve como base o modelo de Gauthier et al (1976). Foi aplicado como piloto na área de Lavanderia do Hospital de Clínicas da UNICAMP.

A eficácia do desempenho hospitalar é avaliada em função de sua prestação de serviços. Os padrões de desempenho da administração do hospital reportam tanto aos aspectos quantitativos como qualitativos desses serviços.

Os padrões referem-se entre outros a: 1. Segurança do Paciente, 2. Resolutividade do atendimento, 3. Vagas necessárias para o atendimento, 4. Qualidade dos serviços profissionais, 5. Tempo de espera para ser atendido.

Como relata Souza (1989) em seu artigo,

[...] o processo de gestão está subordinado à prática dominante em determinado período [...] cada época domina um tipo de política de interesse diverso da do outro, identificando as características dos vários modelos possíveis de gestão e suas diferenças de qualidade (SOUZA, 1989, p. 10-1).

O que implica em: adequação dos atos médicos, inserção de novas tecnologias, qualificação dos recursos humanos, que são agentes principais de mudanças. Como o processo de gestão não se realiza sem a presença do potencial humano, a "gestão de recursos humanos" se dá como num círculo vicioso, assim definido por Dussault (1994, p. 2), tendo de um lado os usuários do sistema e do outro dos governos, nas três esferas (municipal, estadual e federal) que querem mais eficiência no uso dos recursos liberados 
para os serviços de saúde e dos prestadores que querem condições de trabalho mais favoráveis. Os vários recursos segundo Dussault (1994), para produzir bens e serviços destinados a criar um impacto sobre o nível de saúde dos indivíduos, são: cognitivo (saber, tecnologia), físico (edifícios, materiais, suprimentos, etc.), financeiro e humano.

As principais dimensões dessa variação da qualidade dos serviços de Saúde podem ser descritas, segundo Dussault (1994) a seguir:

1. Dimensões técnicas, que dizem respeito à eficácia do serviço (respeito às normas profissionais decorrentes do exercício profissional, às normas técnicas em geral, além das relativas ao uso de equipamentos de proteção individual - EPI);

2. Dimensões de relacionamento referem-se à relação entre os prestadores e os usuários (Pode-se medir a qualidade em termos de satisfação dos usuários, do caráter ético da prestação dos serviços, de sua continuidade, da empatia ou não manifestado e do aspecto de adaptação do serviço);

3. Dimensões econômicas, diz respeito à eficiência (tanto uma melhor relação custobenefício, como um custo aceitável), à produtividade (que diz respeito à utilização da capacidade de produção dos recursos) e a acessibilidade econômica.

A Gestão de Qualidade no serviço público se faz mediante uma adequabilidade e aplicabilidade dos princípios de Deming, em especial, no que se refere à aplicação de conceitos estatísticos. Também é essencial a aplicação da metodologia de Ishikawa para a elaboração da adaptação deste modelo. A importância da Gestão de Qualidade se dá na elaboração e criação de indicadores que venham apoiar decisões administrativas em menor espaço de tempo.

O século passado registrou acontecimentos que modificaram as ações, valores, a cultura, os costumes, os paradigmas até então existentes, lançando o homem ao encontro de um novo horizonte. A própria Administração, como ciência, passa por grandes transformações, passando as metas a serem alcançadas tais como: a melhoria da prestação de serviços ou a melhoria da qualidade da própria prestação de serviços, redução de preços, a limitação à essencialidade e terceirização das atividades entre tantas outras mudanças. 
Com o aparecimento do microcomputador, muitas informações passaram a ser obtidas em menor espaço de tempo. Os relatórios assim emitidos, com a ajuda dessa nova ferramenta, vêem a auxiliar as tomadas de decisões.

Os relatórios deverão contemplar, em sua essência, segundo Morana (1996, p. 114), dada a importância e relevância que passam a ter, os itens abaixo relacionados:

1. Indicadores de crescimento,

2. Evolução da produção e dos custos,

3. Os meios para obter os resultados;

4. A produtividade decorrente dos resultados obtidos em relação aos meios.

O HC é um hospital estadual universitário especializado, nível terciário, com tripla função: Assistência, Ensino e Pesquisa. Traz em sua prática diária, a aplicação das normas e portarias emitidas pelo Ministério da Saúde, além de incorporar conceitos de qualidade, introduzidos pelo Programa da Qualidade no Serviço Público. O Hospital de Clínicas tem capacidade para 403 leitos, incluindo leitos de Unidade de Terapia Intensiva (U.T.I.) Adulto em número de 15, Unidade Coronariana com 05 e Unidade de Terapia Intensiva (U.T.I.) Pediátrica, com 10 leitos.

Pelas suas características de hospital regional, tem em sua clientela, pacientes provenientes em sua maioria da macrorregião de Campinas, DIR XII (Diretoria Regional de Saúde - XII)/Campinas em especial, além seguido da DIR XV - Piracicaba e DIR XX São João da Boa Vista.

\section{OBJETIVOS}

Como diretriz, consiste a priori de uma análise dos conceitos de Deming (1990), Ishikawa (1997) e de uma aplicabilidade dos mesmos num relatório de desempenho identificado por Gauthier et al (1976), de tal forma a se poder fazer uma adaptação num hospital universitário com características próprias.

Os principais objetivos foram:

1. Gerar instrumentos de mensuração, de avaliação e controle dos serviços hospitalares que contivesse como parâmetros de estudo entre outros, um item relativo aos 
custos, além dos relativos à produção e os meios de obtê-la, a fim de fornecer subsídios para tomada de decisões junto a Administração do H.C.;

2. Estabelecer um critério permanente de análise e avaliação de serviços;

3. Criar um modelo de relatório gerencial padrão para os diferentes serviços;

4. Munir os diferentes serviços hospitalares de instrumentos de gerenciamento.

Com esses propósitos serão contempladas as avaliações que a própria área faz de si, bem como as que a Administração Superior faz do desempenho das mesmas, de tal forma que esses relatórios se tornem fonte de informações e de apoio às decisões gerenciais. Segundo o próprio Gauthier et al (1976), um relatório de desempenho deverá ter objetivos definidos pelas próprias áreas e que atendam às necessidades de gerenciamento das mesmas e da própria administração superior.

\section{METODOLOGIA}

A “Análise de Desempenho" como adotada por Gauthier et al (1976), é como um

[...] conjunto de técnicas que têm por objetivo a apreensão da realidade e avaliação da eficácia, mas é também, e na mesma proporção, o estabelecimento de um estado de espírito e clima favoráveis (GAUTHIER et al, 1976, p. 5).

Alia-se a esta noção, os conceitos de Deming (1990), preconizados por Scherkenbach (1992) de melhoria contínua, e os estudos de Sink et al (1993) referentes ao planejamento e medição, para elaborar sistemas de medição que aliem eficiência e eficácia, para o gerenciamento da qualidade e produtividade dos serviços em geral, é que se poderá chegar a uma concepção do que pode ser um relatório de desempenho.

Com a velocidade que as informações atualmente são transmitidas, não utilizar os conceitos acima expostos é ficar à margem dos acontecimentos, é não tomar medidas eficazes a tempo de solucionar problemas gerenciais. Cabe então desenvolver instrumentos de medição, para avaliação e controle de desempenho dos serviços hospitalares, com controle periódico dos resultados, avaliando os motivos pelos os quais os "números" estão aumentando ou diminuindo e avaliar as suas consequiências. Para tal, agregou-se não só conceitos que envolvam a produtividade (relação resultados/recursos), mas conjuntamente conceitos de eficácia e eficiência. Para Sink et al (1993), a produtividade 
[...] é um bom indicador para termos em nosso painel de instrumentos, pois pode dizer-nos, de modo eficiente, o que está acontecendo com o sistema organizacional (SINK et al. 1993, p. 187).

O pensamento que permeia o desenvolvimento deste trabalho é o do Sink et al (1993, p. 163), qual seja: "Não se pode gerenciar aquilo que não se consegue controlar. Daí pode-se deduzir, logicamente, que não se pode gerenciar aquilo que não se pode medir". Conseqüentemente se ter a necessidade de incorporar a medição como ferramenta de melhoria contínua.

O principal objetivo da medição, muitas vezes desprezado, é melhorar sua eficácia, e para isso é necessário dispor de indicadores confiáveis, que representem um indicativo de ações a serem realizadas, quando não priorizadas em busca da eficácia das informações; e que a partir dessa avaliação, se possa verificar a necessidades de melhorias que um processo ou uma atividade são executados.

Para Novaes, citado por Malik et al (1998, p. 46) "Indicadores são medidas quantitativas de qualidade relacionadas à estrutura, processo e resultado da atenção médica". Ainda segundo Malik et al (1998), os

Indicadores muitas vezes são apresentados sob a forma de taxas, e existe um intervalo definido para a vigilância de cada indicador.[...]

Para Laurenti e colaboradores, há pré-requisitos a observar quando da construção e seleção de indicadores:

- $\quad$ Existência e disponibilidade de dados;

- Definições e procedimentos empregados para construir esses indicadores devem ser conhecidos, de modo a possibilitar comparação entre localidades ou organizações diferentes, ou na mesma, em períodos distintos;

- Construção fácil, interpretação simples;

- Reflexo do maior número possível de fatores que reconhecidamente influem no estado de saúde;

- Poder discriminatório, possibilitando comparações”.

(MALIK et al, 1998, p. 47)

A "análise de desempenho", feita através de relatório gerencial, assim definido, alia os parâmetros: produção ou resultados, aos meios ou recursos, custos e produtividade em relação ao que é realizado no mês, no mês anterior, ao acumulado, evolução ao longo do tempo. Demonstrará o perfil dos serviços, a sua inserção num sistema de custos e numa análise última, a sua produtividade.

Que dados são necessários a serem obtidos do sistema a fim de criar informações de apoio, é uma das questões a serem feitas pelo administrador da área. A facilidade de acesso, 
armazenamento, recuperação, custo, disponibilidade e criatividade dos dados são questões a serem tratadas pelos encarregados do projeto e desenvolvimento dos sistemas de medição e avaliação.

A medição é utilizada também para fazer previsões, análise de variações, estimativa de custos, planejamento, avaliação de pessoal, testes, controle de qualidade, controle de produção, elaboração de cronogramas, gerenciamento de projetos, análise e controle orçamentário, decisões sobre investimentos de capital, análise de custo/benefício, análise econômica de engenharia.

A análise de desempenho de um relatório, desta forma, deverá conter elementos essenciais: 1. Apreender a totalidade da atividade da instituição; 2. Proporcionar a facilidade de manuseio da informação, de forma a fornecer subsídios para ações a serem tomadas, além de permitir comparações com as previsões estabelecidas.

Ao se definir as áreas em estudo, neste trabalho, deverá haver um plano de controle, propriamente dito, qual seja, estabelecer os elementos que caracterizam as áreas, elaborar e adaptar a informação percebida na estrutura concebida para o relatório, que por si só já é uma análise de processo.

Em uma atividade muitos processos estão inter-relacionados e o produto ou serviço resultante de um processo pode ser o meio utilizado para outro serviço. Segundo Scherkenbach (1992),

[...] os inputs de um sistema de processamento podem vir dos resultados de um ou vários sistemas de processamento. Esses resultados podem ser faturas, tempo de espera para serviço, um eixo excêntrico, mudanças de projetos de engenharia, um funcionário treinado, ou uma variedade de outras possibilidades (SCHERKENBACH, 1992, p. 19).

Para se ter o conhecimento de um processo, se ele está funcionando de forma correta, é necessário conhecer o que o está influenciando, e o diagrama de Ishikawa, de causa e efeito é uma metodologia apropriada para este momento, pois estuda os componentes que interferem num processo. Daí ele se constituir de importante metodologia no momento.

Em particular neste trabalho, que é o de construir um relatório de desempenho das áreas hospitalares, o diagrama de Ishikawa traz os elementos necessários para uma análise dos indicadores que nele deverão constar, por estar incorporado em sua estrutura a relação 
de causa e efeito, ou seja os 5 M's: mão de obra, máquinas, materiais, métodos e medições e que adaptado para o modelo criado ficará como: produção, meios (recursos humanos) e equipamentos ou o que é específico de cada área, caso haja necessidade, medições, custos, produtividade.

Desta forma cada elemento ou "M" se constituirá de um tipo de indicador, sendo que em futuro, este relatório poderá evoluir para a incorporação de um sistema de gerenciamento financeiro, ou seja, além do custo, agregar os elementos: receita, que no caso será faturamento, e lucro, embora o hospital em estudo não vise o lucro, mas que para outros hospitais particulares será relevante a informação a ser acrescentada.

Pode ter-se a relação receita/faturamento e custo, ou a relação Receita/Despesa, além da relação convencional, que é a relação entre o que se produz, ou seja, produção, e o que ela utilizou para obter a produção e o que ela consome.

A relação faturamento/custos tem a vantagem de levar em conta todos os fatores internos da instituição (consumo de materiais, de energia, equipamentos, horas trabalhadas, etc.), inclui o cliente interno como "fator decisivo de produtividade". O responsável da área passa a ser, o fator decisivo de produtividade, ao gerenciar a receita, caso a ele seja dado um limite do que se é possível produzir e o custo de sua atividade, evitando perdas, retrabalhos, horas extras e outros.

De outra forma, o custo poderá ser expandido em seus diversos elementos e deverá conter também o Diagrama de Ishikawa, de maneira a tornar a análise da área mais completa, favorecendo com a individualização dos elementos que o constitui, maior acuricidade e inferência sobre os resultados, já que um estudo analítico tem por objetivo descobrir as causas de um problema, que poderá ser, por exemplo, descobrir o que está causando um custo alto para uma baixa produção, ou verificar se o fator que determinou o custo foi simplesmente a variação monetária, aumento do preço de insumos por mudança de forma de processamento entre outros.

Para Deming, segundo Scherkenbach (1992),

O objetivo de um estudo estatístico é fornecer uma base para a ação. Num estudo enumerativo, a ação será tomada sobre o material que denominamos universo, que cobre adequadamente o quadro a ser estudado. Somente um conhecimento substantivo (engenharia, psicologia, medicina, agricultura, pesquisa de consumo) pode preencher a lacuna entre o quadro e o universo, quando estes elementos não forem idênticos. 
Num estudo analítico, ação será tomada com base num sistema ou processo de causa, com a esperança de mudar o produto futuro (que poderia ser pessoas, produtos manufaturados ou produtos agrícolas). Resumidamente, um objetivo enumerativo significa contar ou avaliar alguma coisa. Os usos que podem ser feitos de um estudo enumerativo dependem somente da avaliação de um quadro específico (DEMING apud SCHERKENBACH, 1992, p. 97).

Particularmente, para o desenvolvimento da formação do relatório gerencial, será feito um diagnóstico da situação para cada área operacional. Esse diagnóstico envolve um levantamento de uma quantidade de hipóteses, seja feito através do uso de um brainstorming ou de pesquisas, o que virá ampliar o escopo da investigação. A utilidade do diagnóstico será a de identificar o que é realmente importante colocar num relatório específico da área, no que tange em particular a sua atividade, o resultado de uma atividade.

As informações estatísticas-econômicas que constarão do relatório gerencial ou de desempenho devem conter as 4 categorias de indicadores:

1. Indicadores de atividades (produção/resultados): É a própria produção do Serviço, com os recursos que ele possui. No caso da área piloto ser a Lavanderia, ter-se-á os itens e subitens conforme a especificação dos mesmos:

\section{Costura:}

1.1 Confeccionada

1.1.1 Porte I: Tempo de Confecção: 0 a 30 minutos

1.1.2 Porte II: Tempo de Confecção: 31 a 60 minutos

1.1.3 Porte III: Tempo de Confecção: 61 a 120 minutos

1.2. Consertada (Porte I): Tempo de Conserto: de 0 a 30 minutos

2. Roupa Lavada por $(\mathrm{kg})$, divididas em duas áreas:

2.1 Áreas internas do $\mathrm{HC}$,

2.2 Áreas externas rotineiras: Refere-se à prestação de serviço “as áreas suporte/adjacências pertencentes e/ou vínculos com a Universidade”.

2. Indicadores de Meios (recursos): É considerado tudo aquilo que é utilizado para a obtenção dos resultados, para a melhoria ou incremento da produtividade, e deve-se buscar a sua minimização. 
Podem ser tanto recursos humanos ou físicos, e deverão conter informações relativas a pessoal em atividade na área: freqüência, faltas, férias, horas-extras e índice de absenteísmo, para depois num momento seguinte identificar as causas do absenteísmo, se foram por doenças ocupacionais ou não, a fim de criar meios de preveni-las.

Com relação aos funcionários tem-se a considerar alguns conceitos para o cálculo:

(1) Efetivo Atuante: Conterá todos os funcionários em atividade na Lavanderia. Efetivo Atuante: Total de Pessoal Existente - Funcionários Faltantes/Afastamentos,

(2) Índice de Efetivo Atuante:

$$
\left[\left(\frac{\text { Total de Pessoal Existente - Funcionários Faltantes }}{\text { Total de Pessoal Existente }}\right) \times 100 \%\right] \text {, }
$$

(3) Índice Absenteísmo: 100\% - Índice de Efetivo Atuante,

Observa-se que com este índice, algumas hipóteses poderão ser levantadas, como, por exemplo, se existe um programa de educação continuada que sirva como prevenção não só de doenças ocupacionais, mas de manuseio das próprias ferramentas de trabalho?

3. Indicadores de Custo: Estudar o custo ao longo do tempo, o que o está influenciando. Estudar também qual custo está aumentando ou diminuindo. O método a ser utilizado, passada a etapa preliminar, será o de separar os custos fixos ou de estrutura, dos custos variáveis ou operacionais.

A análise dos custos deverá abranger em futuro, três subdivisões discriminadas a seguir:

3.1 Estudo de quadro de pessoal, sob a ótica das horas-extras (caso haja), doenças ocupacionais no que se refere a tratamento preventivo. Pode estar havendo um custo muito significativo, por exemplo, em contrato de horas extras, quando o custo seria menor se contratar um funcionário que permaneça no próprio serviço,

3.2 Estudo dos equipamentos, que envolverá depreciação dos mesmos, questões do tipo: que tipos de manutenção estão sendo feitos nos mesmos (se corretiva ou preventiva). Avaliar a situação dos equipamentos, ou seja, verificar se estão obsoletos, e, se por causa disso, o custo está sendo alto com manutenção. 
3.3 Estudo dos materiais: questões relativas a previsão de compras, fornecedores (o padrão de qualidade da compra).

Os indicadores de custos propiciarão em última instância, com o estudo ao longo do tempo, encontrar fatores que estão influenciando os mesmos, que determinarão a inclinação da curva, e estudar qual custo está aumentando ou diminuindo.

4. Indicadores de Produtividade. Será uma relação decorrente dos resultados obtidos em relação aos meios.

$$
\text { Produtividade }=\frac{\text { Produção }}{\text { Meios }} \quad(\text { Recursos Humanos em atividade na área })
$$

Produtividade é um importante critério de desempenho porque, quando se mede bem, acaba-se aprendendo algo sobre eficácia, eficiência e qualidade. Nesse sentido, ela é uma boa medida de diagnóstico.

É na verdade, um macro-indicador que se refere à relação entre Resultados e Recursos.

Recursos: É tudo aquilo que é utilizado para a obtenção dos resultados. Deve-se buscar a sua minimização.

Resultados: É tudo aquilo que tiver no final de um processo.

Então:

Produtividade $=\frac{\text { Resultados }}{\text { Recursos }}$ Deve-se buscar a maximização de resultados.

O que é intrínseco ao conceito de Produtividade:

- Produzir cada vez mais e/ou melhor, com cada vez menos. Pode-se, pois, representar a produtividade como o quociente entre o que a instituição produz e o que ela consumiu na produção;

- Agregar o máximo de valor ao menor custo.

Segundo Sink et al (1993), não basta aumentar a quantidade produzida, é necessário que o produto tenha valor, que atenda às necessidades dos clientes. Pois quanto 
maior a produtividade de uma empresa, mais útil ela é para a sociedade, pois está atendendo às necessidades dos seus clientes a um baixo custo.

Dessa forma, a Produtividade poderá ser também as seguintes relações:

Produtividade $=\frac{\text { "Qualidade" }}{\text { "Custos" }}$ ou Produtividade $=\frac{\text { "ValorProduzido" }}{\text { "ValorConsumido" }}$

Esta afirmação de produtividade torna clara a afirmação de Deming, segundo Sherkenbach (1992) de que a produtividade é aumentada pela melhoria da qualidade.

Portanto, considerando-se apenas os fatores internos de uma organização, a produtividade só pode ser aumentada pelo aporte de capital e pelo aporte de conhecimento.

No caso em estudo, na Lavanderia, a Produtividade será vista sob os pontos de vista como:

$$
=\frac{\text { "Peças" }}{\text { "Funcionáro da Costura" }},=\frac{\text { "Kg roupa lavada" }}{\text { "Funcionário Operacional" }}
$$

Como a área piloto foi a Lavanderia em trabalho realizado (ARANHA; LEICHSENRING; MADEIRA, 1997), num primeiro instante, constituiu-se em fazer um diagnóstico da situação, detectou-se os seus problemas específicos quanto aos quesitos: quadro de pessoal, produção, custos, produtividade de maneira geral e perfil de maquinário. A partir daí foi criado um quadro ou tabela de referência que se convencionou chamar "indicadores de desempenho". 


\section{RELATÓRIO GERENCIAL}

PERÍODO: JUL/97

\begin{tabular}{|c|c|c|c|c|c|}
\hline \multicolumn{2}{|l|}{ LAVANDERIA } & \multicolumn{3}{|c|}{ DIRETORIA: } & RAMAL: \\
\hline INDICADORES & $\begin{array}{l}\text { REALIZADAS } \\
\text { (MÊS:JULHO) }\end{array}$ & $\begin{array}{c}\text { REALIZADAS } \\
\text { (M. } \\
\text { ANTERIOR) } \\
\end{array}$ & $\begin{array}{l}\text { ACUMULADO } \\
\text { (Jan a Julho) }\end{array}$ & $\begin{array}{l}\text { MÉDIA } \\
\text { DIÁRIA } \\
\text { (30 DIAS) } \\
\end{array}$ & EVOLUÇÃO \\
\hline
\end{tabular}

\section{1 - RESULTADOS}

\section{No. ROUPAS (peças)}

1. Costura

\begin{tabular}{|l|r|r|r|r|r|}
\hline 1.1 Confeccionada & $\mathbf{3 . 5 9 6}$ & $\mathbf{2 . 0 2 3}$ & $\mathbf{1 9 . 0 4 3}$ & $\mathbf{1 1 9 , 8 7}$ & $\mathbf{7 7 , 7 6 \%}$ \\
\hline 1.1 .1 Porte I & 3.402 & 1.891 & 18.205 & 113,40 & $79,90 \%$ \\
\hline 1.1 .2 Porte II & 126 & 96 & 680 & 4,20 & $31,25 \%$ \\
\hline 1.1 .3 Porte III & 68 & 36 & 158 & 2,27 & $88,89 \%$ \\
\hline 1.2 Consertada (Porte I) & $\mathbf{4 . 1 0 0}$ & $\mathbf{4 . 1 7 9}$ & $\mathbf{2 9 . 4 0 6}$ & $\mathbf{1 3 6 , 6 7}$ & $-1,89 \%$ \\
\hline 1.3 Destruição & $\mathbf{9 2 7}$ & $\mathbf{1 . 0 1 6}$ & $\mathbf{7 . 3 5 2}$ & $\mathbf{3 0 , 9 0}$ & $-8,76 \%$ \\
\hline
\end{tabular}

\section{Lavada (kg)}

2.1 Áreas Internas

2.1.1 NAMC

2.1.2 PEDIATRIA

2.1.3 EE/CT

2.1.4 U.T.I./U.RESP.

2.1.5 P.S.

2.1.6 C.CIRÚRGICOS

2.1.7 AMBULATÓRIOS

2.1.8 PROCS.ESPS.

2.1.9 RADIOLOGIA:

$*$ RAIO X

* ULTRASSONOG.

* RADIOTERAPIA

* MEDICINA NUCLEAR

2.1.10 LABORATÓRIOS

2.1.11 COORD. ASSIST.

2.1.12 DND:

* SONDAS

* LACTÁRIO

* OUTRAS

2.1.17 CENTRAL MAT.

2.1.18 CCI

2.1.19 CAT.CARDÍACO

\begin{tabular}{|c|c|}
\hline 107.272 & 102.248 \\
\hline 101.477 & 95.529 \\
\hline 26.141 & 24.407 \\
\hline 6.328 & 5.960 \\
\hline 4.856 & 4.79 \\
\hline 4.515 & 4.5 \\
\hline 8.471 & 8.779 \\
\hline 16.207 & 14.423 \\
\hline 2.601 & 2.38 \\
\hline 2.126 & 2.325 \\
\hline 2.404 & 2.343 \\
\hline 1.099 & 1.136 \\
\hline 825 & 842 \\
\hline 145 & 104 \\
\hline 335 & 262 \\
\hline 94 & 130 \\
\hline 1.109 & 1.324 \\
\hline 964 & 1.077 \\
\hline 231 & 252 \\
\hline 669 & 761 \\
\hline 64 & 64 \\
\hline 22.863 & 20.061 \\
\hline 125 & 10 \\
\hline 270 & \\
\hline
\end{tabular}

\begin{tabular}{|r|r|r|}
$\mathbf{8 0 5 . 6 9 7}$ & $\mathbf{3 5 7 5 , 7 5}$ & $\mathbf{4 , 9 1 \%}$ \\
\hline $\mathbf{4 8 2 . 8 9 7}$ & $\mathbf{3 3 8 2 , 5 8}$ & $\mathbf{6 , 2 3 \%}$ \\
\hline 166.953 & 871,37 & $7,11 \%$ \\
\hline 44.724 & 210,95 & $6,19 \%$ \\
\hline 34.150 & 161,85 & $1,22 \%$ \\
\hline 28.650 & 150,48 & $-1,09 \%$ \\
\hline 56.779 & 282,35 & $-3,51 \%$ \\
\hline 99.604 & 540,22 & $12,37 \%$ \\
\hline 15.974 & 86,70 & $9,83 \%$ \\
\hline 14.712 & 70,88 & $-8,55 \%$ \\
\hline 14.686 & 80,15 & $2,63 \%$ \\
\hline 6.588 & 36,65 & $-3,19 \%$ \\
\hline 5.291 & 27,49 & $-2,08 \%$ \\
\hline 872 & 4,84 & $40,12 \%$ \\
\hline 1.935 & 11,18 & $28,24 \%$ \\
\hline 662 & 3,13 & $-27,58 \%$ \\
\hline 9.947 & 36,95 & $-16,27 \%$ \\
\hline 6.546 & 32,14 & $-10,48 \%$ \\
\hline 1.665 & 7,71 & $-8,31 \%$ \\
\hline 4.623 & 22,29 & $-12,08 \%$ \\
\hline 258 & 2,13 & $0,00 \%$ \\
\hline 142.869 & 762,08 & $13,96 \%$ \\
\hline 597 & 4,16 & $17,36 \%$ \\
\hline 1.750 & 9,00 & $-45,74 \%$ \\
\hline & &
\end{tabular}

Serviço Social \& Saúde, Campinas, v.2, n.2, p. 83-102 


\begin{tabular}{|c|c|c|c|c|c|}
\hline INDICADORES & REALIZADAS & $\begin{array}{c}\text { REALIZADAS } \\
\text { (M. } \\
\text { ANTERIOR) }\end{array}$ & $\begin{array}{c}\text { ACUMULADO } \\
\text { (Jan a Julho) }\end{array}$ & $\begin{array}{c}\text { MÉDIA } \\
\text { DIÁRIA } \\
\text { (30 DIAS) }\end{array}$ & EVOLUÇÃO \\
\hline
\end{tabular}

\begin{tabular}{|l|r|r|r|r|r|}
\hline 2.2 Áreas Ext. Rotineiras & $\mathbf{5 . 7 6 4}$ & $\mathbf{6 . 6 9 0}$ & $\mathbf{1 5 2 . 1 4 8}$ & $\mathbf{1 9 2 , 1 3}$ & $\mathbf{- 1 3 , 8 4 \%}$ \\
\hline 2.1 .1 CECOM & 381 & 361 & 2.201 & 12,70 & $5,54 \%$ \\
\hline 2.1 .2 CIPOI & 31 & 29 & 134 & 1,03 & $6,90 \%$ \\
\hline 2.1 .3 F.C.M. & 78 & 126 & 822 & 2,60 & $-38,10 \%$ \\
\hline 2.1 .4 GASTROCEN. & 805 & 492 & 4.510 & 26,83 & $63,62 \%$ \\
\hline 2.1 .5 HEMOCENTRO & 156 & 170 & 696 & 5,20 & $-8,24 \%$ \\
\hline 2.1 .6 CAISM & 2.799 & 3.715 & 132.586 & 93,30 & $-24,66 \%$ \\
\hline 2.1 .7 FAC.ODONT. & 47 & 97 & 297 & 1,57 & $-51,55 \%$ \\
\hline 2.1 .8 SOC.BRAS.FACE & 149 & 388 & 1.693 & 4,97 & $-61,60 \%$ \\
\hline 2.1.9 REITORIA & 84 & 79 & 526 & 2,80 & $6,33 \%$ \\
\hline 2.1 .10 CRECHE A.SAUDE & 1.234 & 1.233 & 8.683 & 41,13 & $0,08 \%$ \\
\hline
\end{tabular}

\section{2-RECURSOS}

\begin{tabular}{|l|r|r|r|r|r|}
\hline .FUNC./HC/FUNC. & $\mathbf{8 5}$ & $\mathbf{8 4}$ & $\mathbf{8 4}$ & $\mathbf{8 4}$ & $\mathbf{1 , 1 9 \%}$ \\
\hline .Pes. Chefia(Diretoria) & 1 & 1 & 1 & 1 & $0,00 \%$ \\
\hline .Pes.Adm.(Secretária) & 1 & 1 & 1 & 1 & $0,00 \%$ \\
\hline .Pes.Chefia(Proces.) & 6 & 6 & 6 & 6 & $0,00 \%$ \\
\hline .Pes. Oper.1(Proces.) & 57 & 58 & 57 & 57 & $-1,72 \%$ \\
\hline .Pes. Chefia(Costura) & 1 & 1 & 1 & 1 & $0,00 \%$ \\
\hline .Pes. Oper.(Costura) & 2 & 2 & 2 & 2 & $0,00 \%$ \\
\hline .FUNC.COM RESTRIÇÕES & 12 & 13 & 12 & 12 & $-7,69 \%$ \\
\hline .FUNC.RETORNO INSS & 0 & 1 & 0 & 0 & $-100,00 \%$ \\
\hline .FUNC.AFASTASTADOS-INSS & 0 & 0 & 0 & 0 & INDETERM. \\
\hline .FUNC. ADMITIDO & 3 & 0 & 3 & 3 & INDETERM. \\
\hline .FUNC.C/AUS.LEGAL & 1 & 1 & 1 & 1 & $0,00 \%$ \\
\hline
\end{tabular}

\begin{tabular}{|l|r|r|r|r|r|}
\hline SAÍDA & & & & & \\
\hline .FUNC. DEMITIDO & 0 & 1 & 0 & 0 & $-100,00 \%$ \\
\hline .FUNC. TRANSFERIDO & 1 & 0 & 1 & 1 & INDETERM. \\
\hline
\end{tabular}

\section{. EFETIVO ATUANTE}

AFASTAMENTOS DIAS:

\section{.FUNC.}

FALTAS/AFASTAMENTOS

.FUNC. AUSÊNCIAS LEGAIS

.IND. EFETIVO ATUANTE

\begin{tabular}{|l}
\hline \\
\hline .IND.ABSENTEÍSMO:
\end{tabular}

(DO PESSOAL EM ATIVIDADE)

.IND. "ABSENTEÍSMO" :

( RELATIVO ÁS AUSENCIAS

LEGAIS)

\begin{tabular}{|l|r|r|r|r|}
84 & 83 & 83 & 83 & $1,20 \%$ \\
\hline
\end{tabular}

\begin{tabular}{|c|c|c|c|c|}
\hline 90 & 117 & 1019 & 3,00 & $-23,08 \%$ \\
\hline 264 & 322 & 1479 & 8,08 & $-18,01 \%$ \\
\hline $96,43 \%$ & $95,30 \%$ & - & - & - \\
\hline $3,57 \%$ & $4,70 \%$ & - & - & - \\
\hline & & & & \\
\hline $10,48 \%$ & $12,93 \%$ & & & \\
\hline & & & & \\
\hline
\end{tabular}




\begin{tabular}{|c|l|l|l|c|c|}
\hline INDICADORES & REALIZADAS & $\begin{array}{l}\text { REALIZADAS } \\
\text { (M. }\end{array}$ & $\begin{array}{l}\text { ACUMULADO } \\
\text { ANTERIOR) }\end{array}$ & $\begin{array}{c}\text { MÉDIA } \\
\text { DIÁRIA } \\
\text { (Jan a Julho) }\end{array}$ & EVOLUÇÃO \\
& (30 DIAS) & \\
\hline
\end{tabular}

HORAS EXTRAS

\begin{tabular}{|l|r|r|r|r|r|}
\hline HORAS EXTRAS & 1.322 & 1.193 & 8.733 & 44,07 & $10,81 \%$ \\
PROCESSAMENTO & 458 & 424 & 3.008 & 15,27 & $8,02 \%$ \\
\hline HORAS EXTRAS COSTURA & & &
\end{tabular}

\section{3 - CUSTOS}

\begin{tabular}{|l|r|r|r|r|l|}
\hline Custo Geral Rouparia(Kg) & $\mathrm{R} \$ 2,16$ & $\mathrm{R} \$ 1,95$ & - & - & - \\
\hline
\end{tabular}

\section{4 - PRODUTIVIDADE}

\begin{tabular}{|l|r|r|r|r|r|}
\hline $\begin{array}{l}\text { Kg. roupa lavada/Funcionário } \\
\text { operacional }\end{array}$ & 1.703 & 1.598 & 7.782 & 56,76 & $6,58 \%$ \\
\hline Peças/Funcionário Costura & 2.565 & 2.067 & 11.180 & 85,51 & $24,09 \%$ \\
\hline
\end{tabular}

FONTE: Serviço de Estatística/H.C./UNICAMP

NOTA: Índice de Efetivo Atuante: todos os funcionários no processamento e costura. 


\section{RESULTADOS}

Levando em consideração a especificidade do serviço, foram levantados os itens: a produção total, o efetivo atuante de funcionários, o custo e a produtividade e elaboradas as planilhas: "perfil de máquinas" e "controle de roupas por período de uso" para a Lavanderia. Buscando sistematizar as informações, foi criado um sintético "manual de orientação" com as normas para preenchimento dos relatórios, de tal forma que se evitasse dúvidas no levantamento de dados, bem como fornecesse material de apoio para futuras pessoas que viessem a assumir os serviços, ou mesmo até na ausência das mesmas.

Como a finalidade é fornecer para a gerência direta bem como para a própria Administração do Hospital, elementos para avaliação dos serviços prestados tanto para a comunidade interna como externa do hospital, o modelo de relatório gerencial preenche ao que ele se propôs: através de criação de instrumentos de medição, avaliar permanentemente o desempenho dos serviços hospitalares através do que se pode constituir um relatório padrão para os diferentes serviços hospitalares.

\section{CONCLUSÕES:}

Este estudo possibilitou conhecer melhor a realidade da área abordada, detectar problemas ou necessidades e contribuir para uma possível avaliação gerencial.

Considera-se que este modelo de relatório gerencial, que é um modelo de desempenho para áreas hospitalares, poderá: (1) Fornecer elementos que subsidiem a tomada de decisões para a melhoria e aproveitamento dos recursos humanos e materiais; (2) Favorecer um melhor gerenciamento do custo, no desenvolver das atividades administrativas das chefias e pela Administração do Hospital em especial;

(3) Auxiliar na busca de tipos intervenções que deverão ser feitas para melhorar os desempenhos das áreas e qual a melhor forma para abordar os problemas existentes; (4) Utilizar a metodologia do Diagrama de Ishikawa, a qual tornou possível atingir os objetivos propostos dentro da busca de soluções dos problemas, na otimização dos recursos existentes, na viabilidade de um relatório gerencial que pode ser desenvolvido para as demais áreas hospitalares, sejam elas assistenciais, operacionais e administrativas; (5) Encontrar um bom sistema de medições, baseado em metodologias estatísticas, apropriadas para aplicação neste estudo. O que poderá vir propiciar novas investigações futuras, novas 
inferências neste modelo, a fim de ampliá-lo e adaptá-lo novamente as necessidades do hospital, frente a novos questionamentos, a novas soluções na busca da melhoria contínua.

\begin{abstract}
ABSTRAT
The Study descriptive of a model of Managerial Report, that was elaborated for the area of Laundry of the Hospital of Clinics (HC) of UNICAMP with the purpose of evaluating the acting of the activities accomplished by this area. For such an attainment they were applied the generic instruments, starting from statistical techniques, with base in the model of Gauthier and in the ideas extolled by Deming, Sink, with views to guarantee the effectiveness of the system of information, through indicators that allow the mensuration and control, for the improvement of the performance of those services from hospital. Taking in consideration the specific of the service, it was lifted up, the total production and elaborated the spreadsheets: "profile of machines" and "control of clothes for use period" to the Laundry. Looking for to systematize the information, a synthetic one was created "orientation manual" with the norms for completion of the report. This study made possible to know the reality of the approached area better, to detect problems or needs and to contribute for the managerial evaluation. Its is considered that this model can supply elements that subsidize the electric outlet of decisions for the improvement and use of the human resources and materials, in developing of the administrative activities of the leaderships and for the Administration of the Hospital.
\end{abstract}

KEYWORDS: Indicative of Health; Managerial Report; Health Statistics

\title{
REFERÊNCIAS BIBLIOGRÁFICAS
}

ARANHA, G. T. C. et al. Adaptação de um modelo de relatório gerencial, para avaliação de serviços hospitalares. Caderno de Resumos. I Simpósio de Técnicos de Apoio ao Ensino e à Pesquisa, Campinas, UNICAMP, 1997, 163 p.

AZEVEDO, A. C. Indicadores de qualidade e produtividade em serviços da saúde. Revista Indicadores da Qualidade e Produtividade, São Paulo, v.1, 1993, p. 48-55. 
DEMING, W. E. Qualidade: A revolução da administração. Rio de Janeiro, MarquesSaraiva, 1990.

DUSSAULT, G. Gestão dos recursos humanos e qualidade dos serviços de saúde. Educación Médica y Salud, v. 28, $n^{\circ}$ 4, 1994, p. 478-489.

GAUTHIER, P. H. et al. Análise do desempenho hospitalar. CEDAS, São Paulo, 1976, $111 \mathrm{p}$.

GAUTHIER, P. H. et al. O controle de gestão no hospital. CEDAS, São Paulo, 1979, 218p. GOUVÊA, C.S.D. et al. Produção de serviços e qualidade da assistência hospitalar no Estado do Rio de Janeiro. Revista Saúde Pública, São Paulo, v.31; cap. 6, 1997, p. 601$17 \mathrm{p}$.

ISHIKAWA, K. Controle de Qualidade Total. Rio de Janeiro, Campus, 1997.

MALIK, A. M. et al. Qualidade na gestão local de serviços e ações de Saúde. Revista Saúde e Cidadania, São Paulo, v. 3, 1998, 233 p.

MINAYO, M.C. O desafio do conhecimento. São Paulo - Rio de Janeiro, Hucitec Abrasco, $6^{\text {a }}$ ed., 1999, 255 p.

MORAES, I.H.S. Informações em saúde: da prática fragmentada ao exercício de cidadania. São Paulo - Rio de Janeiro, Hucitec-Abrasco, 1994, 172 p.

MORANA, S. Administração a custo zero. São Paulo, STS Publicações e Serviços LTDA. $3^{\text {a }}$ ed., 1996, 203 p.

MOURA. E. As sete ferramentas gerenciais da qualidade. Implementando a melhoria contínua com maior eficácia. Makronbooks, São Paulo, 1994, 118p.

FIO CRUZ. 1997 O Ano da Saúde. Entre o Estado Mínimo e o Estado Necessário. Rio de Janeiro, RADIS, nº14, jul de 1997, p. 3-6.

SANTOS, N.R. Governabilidade na Saúde. São Paulo, Saúde em Debate, v.34, março, 1992, 4-12p.

SINK, D. S. et al. Planejamento e medição para a performance. Rio de Janeiro, Qualitymark, 1993, 343 p.

SCHERKENBACH, W. W. O Caminho de Deming para a qualidade e produtividade. Qualitymark, $5^{\text {a }}$ reimpressão, Rio de Janeiro, 1992, 149 p.

SOUZA, G.W. Planejamento sem normas. São Paulo, Hucitec, 1989. 143 p. 
ZANON, U. Qualidade da assistência médica hospitalar, Revista Administração da Saúde, São Paulo, v.2, nº5, Jul/Set de 2000, p. 15-22. 